\title{
ON FINITE $p$-GROUPS CONTAINING A MAXIMAL ELEMENTARY ABELIAN SUBGROUP OF ORDER $p^{2}$
}

\author{
YAKOV BERKOVICH \\ University of Haifa, Israel
}

\begin{abstract}
We continue investigation of a $p$-group $G$ containing a maximal elementary abelian subgroup $R$ of order $p^{2}, p>2$, initiated by Glauberman and Mazza $[\mathrm{GM}]$; case $p=2$ also considered. We study the structure of the centralizer of $R$ in $G$. This reduces the investigation of the structure of $G$ to results of Blackburn and Janko (see references). Minimal nonabelian subgroups play important role in proofs of Theorems 2 and 5 .
\end{abstract}

Glauberman and Mazza ([GM]) have proved that if a $p$-group $G, p>2$, possesses a maximal elementary abelian subgroup $R$ of order $p^{2}$ (i.e., $R$ is not contained in an elementary abelian subgroup of $G$ of order $p^{3}$ ), then $G$ has no elementary abelian subgroup of order $p^{p+1}$. The proof of this deep result is not elementary.

In this note we continue to study the structure of groups from [GM] clearing the structure of $\mathrm{C}_{G}(R)$ also in case $p=2$. In the last case, there is $G$ containing an elementary abelian subgroup of order $2^{4}$, and all such $G$ are classified in [BJ1, Theorem 127.1].

We use elementary prerequisites only and standard notation (see [BJ1, $\mathrm{BJ} 2, \mathrm{~B}]$.$) Only finite p$-groups are considered, $p$ is a prime. By $\mathrm{C}_{p^{n}}, \mathrm{E}_{p^{n}}$, $\mathrm{D}_{2^{n}}$ and $\mathrm{Q}_{2^{n}}$ we denote cyclic, elementary abelian, dihedral and generalized quaternion groups of orders $p^{n}, p^{n}, 2^{n}$ and $2^{n}$, respectively. Next, Z $(G)$ is the center of $G$ and $\Phi(G)$ its Frattini subgroup, $\mathrm{d}(G)=\log _{p}(|G: \Phi(G)|)$.

The N/C-theorem ([B, Introduction, Proposition 12]) asserts that if $H \leq$ $G$, then the number $\left|\mathrm{N}_{G}(H) / \mathrm{C}_{G}(H)\right|$ divides $|\operatorname{Aut}(H)|$.

Let $G$ be a minimal nonabelian $p$-group (see [B, Exercise 1.8a] and [BJ1, Lemma 65.1]). Then (i) $\mathrm{Z}(G)=\Phi(G)$ has index $p^{2}$ in $G$, (ii) $\left|\Omega_{1}(G)\right| \leq p^{3}$

2010 Mathematics Subject Classification. 20D15.

Key words and phrases. Minimal nonabelian p-group, maximal elementary abelian subgroup, soft subgroup. 
and, if $\left|\Omega_{1}(G)\right| \leq p^{2}$, then $G$ is metacyclic. If, in addition, $G$ is metacyclic of order $>p^{3}$, then $G=B \cdot A$, a semidirect product with cyclic kernel $A$ and cyclic complement $B$.

If all minimal nonabelian subgroups of a nonabelian 2-group $G$ are $\cong \mathrm{Q}_{8}$, then $G=Q \times E$, where $Q$ is generalized quaternion and $\exp (E)$ divides 2 ([BJ1, Corollary A.17.3]). This result is used essentially in the proof of Theorem 5.

If a 2-group $G$ has a maximal elementary abelian subgroup $R$ of order 4, then every subgroup of $G$ is generated by four elements so $G$ has no elementary abelian subgroup of order $2^{5}$. Indeed, in view of MacWilliams' theorem (see [BJ1, Theorem 50.3]), it suffices to show that $G$ has no normal elementary abelian subgroup of order 8 . Assume that $E \cong \mathrm{E}_{8}$ is a normal subgroup of $G$. Then, since $R E$ is not of maximal class ([B, Proposition 1.6]), we get $\mathrm{C}_{R E}(R)>R$ ([B, Proposition 1.8]), and $\mathrm{C}_{R E}(R)$ is elementary abelian, by the modular law, and this is a contradiction. Note that the wreath product $G=\mathrm{Q}_{2^{n}}$ wr $\mathrm{C}_{2}$ has a maximal elementary abelian subgroup of order 4 and a maximal subgroup $B$ (the base of this wreath product) with $\mathrm{d}(B)=4$.

We begin with the following

Proposition 1. Suppose that a p-group $G$ contains a maximal elementary abelian subgroup $R$ of order $p^{2}$ and $R$ is not $G$-invariant. If $x \in R-\mathrm{Z}(G)$, then $C_{G}(x)=\mathrm{C}_{G}(R)$ has no metacyclic subgroup of order $p^{4}$ and exponent $p^{2}$.

Proof. By hypothesis, $G$ is nonabelian and $\Omega_{1}(\mathrm{Z}(G))<R$. Write $C=$ $\mathrm{C}_{G}(R)$ and $N=\mathrm{N}_{G}(R)$; then $|N: C|=p$, by the N/C-theorem. Obviously, $\Omega_{1}(C)=R$. Assume, by way of contradiction, that $L \leq C$ is metacyclic of order $p^{4}$ and exponent $p^{2}$. Clearly, $\mathrm{Z}(G) \cap R=U$ has order $p$ (indeed, $\left.R \Omega_{1}(\mathrm{Z}(G))=R\right)$ so $\mathrm{Z}(G)$ is cyclic. If $y \in R-U$, then $\mathrm{C}_{G}(y)=C\left(=\mathrm{C}_{G}(R)\right)$ since $R=\langle y\rangle \times U$ and $U \leq \mathrm{Z}(G)$. Since $\exp (L)=p^{2}$ and $L$ is metacyclic, we have $L=A B$, where $A$ and $B$ are cyclic of order $p^{2}$ such that $A \cap B=\{1\}$. At least one of subgroups $\Omega_{1}(A), \Omega_{1}(B)$ is different from $U\left(=\Omega_{1}(\mathrm{Z}(G))\right)$; denote that subgroup by $\langle x\rangle$; then, as we have noticed, $\mathrm{C}_{G}(x)=C$. Let, for definiteness, $x \in A$. We have $\langle x\rangle \times U=R$.

If $G$ has no normal abelian subgroup of type $(p, p)$, it is a 2-group of maximal class ([B, Lemma 1.4]), and such $G$ has no subgroup isomorphic to $L$, a contradiction. Let $E \triangleleft G$ be abelian of type $(p, p)$; then $R \neq E$, by hypothesis, and $U<E$. Write $F=\langle x, E\rangle$, where $x$ is chosen in the previous paragraph. Clearly, $x \notin E$ (otherwise, $E=\langle x\rangle \times U=R$ ). Since $R<F$ and $\Omega_{1}(F)=F$, it follows that $F$ is nonabelian of order $p^{3}$. Recall that $A<L$ is the cyclic subgroup of order $p^{2}$ containing $x$. In this case, $W=A \cdot E$ is the natural semidirect product with kernel $E$; then $F<W$ and $F / E$ is a unique subgroup of order $p$ in the cyclic group $W / E$ of order $p^{2}$. Since the centralizer $\mathrm{C}_{W}(E)$ has index $\leq p$ in $W$, it contains $F$ so $F$ is abelian, a contradiction. Thus, $L$ does not exist. 
TheOREM 2. Suppose that a nonabelian p-group $G, p>2$, possesses a maximal elementary abelian subgroup $R$ of order $p^{2}$. Set $C=\mathrm{C}_{G}(R)$. Then one of the following holds:

(a) $G$ is metacyclic; then $R=\Omega_{1}(G)$.

(b) $G$ is a p-group of maximal class. If, in addition, $R \triangleleft G$, then $p=3 .^{1}$

(c) $C$ has a cyclic subgroup of index $p$ so it is abelian of type $\left(p^{n}, p\right), n>1$.

Proof. If $R \leq \mathrm{Z}(G)$, then $C=G$ has no elementary abelian subgroup of order $p^{3}$ so it is metacyclic ([Bla1]; see also [B, Theorem 13.7]). In what follows we assume that $R \not Z \mathrm{Z}(G)$; then $\mathrm{Z}(G)$ is cyclic. It follows that, in any case, $C$ is metacyclic.

Suppose that $R \triangleleft G$. If $G$ contains an elementary abelian subgroup $A$ of order $p^{3}$, then $\mathrm{C}_{R A}(R)>R$ is elementary abelian, contrary to the hypothesis. Then, by [B, Theorem 13.7], one of the following holds: (i) $G$ is metacyclic. (ii) $G=\Omega_{1}(G) Z$, where $\Omega_{1}(G)$ is nonabelian of order $p^{3}$ and exponent $p$ and $Z$ is cyclic. (iii) $G$ is a 3 -group of maximal class. In case (i), there are no further restrictions on the structure of $G$. In case (ii), $\mathrm{C}_{G}(R)$ has a cyclic subgroup of index $p$. In case (iii), if $|G|>3^{4}$, we have $R=\Omega_{1}(\Phi(G)$ ) and $\mathrm{C}_{G}(R)=C$ has no cyclic subgroups of index 3, by [B, Exercise 9.1(c)]; in this case, $C$ is either abelian or minimal nonabelian. In what follows we assume that $R$ is not $G$-invariant; then $G$ is not metacyclic. We also assume that $G$ is not of maximal class. Then $|C|>p^{2}$ ([B, Proposition 1.8]).

In that case, by Proposition $1, C$ has no metacyclic subgroup of order $p^{4}$ and exponent $p^{2}$. We claim that then $C$ has a cyclic subgroup of index $p$. One may assume that $|C|>p^{4}$. Since $C$ is regular ([B, Theorem 7.1(c)]) and metacyclic, we have $\left|\Omega_{2}(C)\right| \leq p^{4}$ and $\exp \left(\Omega_{2}(C)\right)=p^{2}$ so $\left|\Omega_{2}(C)\right|=p^{3}$ by what has just been said. It follows that $C / R$ has only one subgroup, namely, $\Omega_{2}(C) / R$, of order $p$, and hence it is cyclic. If $Z<C$ is maximal such that $R \not Z Z(Z$ exists since $R \not \leq \Phi(C))$, then $Z$ is cyclic of index $p$ in $C$. Since $R \leq \mathrm{Z}(C)$, the subgroup $C$ is abelian of type $\left(p^{n}, p\right)$ as in (c).

Note that the $p$-groups $G, p>2$, such that $\mathrm{C}_{G}(x)$ is abelian of type $\left(p^{n}, p\right)$ for some $x \in G$ of order $p$, were studied in great detail in rarely cited important Blackburn's paper [Bla2]; that paper yields essential additional information on groups in part (c) of Theorem 2.

A subgroup $A$ of a $p$-group $G$ is said to be soft in $G$, if $\mathrm{C}_{G}(A)=A$ and $\left|\mathrm{N}_{G}(A): \mathrm{C}_{G}(A)\right|=p([\mathrm{H}])$. Thus, soft subgroups are abelian. A subgroup $C$ of Theorem 2(c) is soft in $G$ as we have noticed in the first paragraph of the proof of Proposition 1. Moreover, if a nonnormal $R<G$ is of order $p^{2}$, then $\left|\mathrm{N}_{G}(R): \mathrm{C}_{G}(R)\right|=p$, and, in addition, $\mathrm{C}_{G}(R)$ is abelian, then it is soft in $G$.

\footnotetext{
${ }^{1}$ If a 3-group $G$ of maximal class is not isomorphic to a Sylow 3-subgroup of the symmetric group of degree $3^{2}$, then all maximal elementary abelian subgroups of $G$ have order $3^{2}$ ([B, Exercise 9.13]). If $p>3$, then there is a $p$-group $G$ of maximal class and order $>p^{4}$ that has no such a subgroup as $R$ (this is a case, if $\Omega_{1}(G) \leq \Phi(G)$ ).
} 
Soft subgroups have a number of remarkable properties (see $[\mathrm{H}]$ and further papers of L. Hethelyi listed in MathSciNet; see also [BJ2, §130]). One of such properties is proved in Remark 3 (note that this proof is distinct from the original one due to L. Hethelyi in $[\mathrm{H}])$.

REMARK 3. (The result of this remark coincides with [BJ2, Lemma 130.2] and taken from $[\mathrm{H}])$. Let $A$ be a nonnormal maximal abelian subgroup of a group $G$ and $\left|\mathrm{N}_{G}(A): A\right|=p$. Let us prove that, if $A<H<G$, then $\left|\mathrm{N}_{G}(H): H\right|=p$ (it follows from this that there is only one maximal chain connecting $A$ with $G$ ). Set $N_{0}=\mathrm{N}_{G}(A)$; then $N_{0}$ is nonabelian. Set $N_{1}=$ $\mathrm{N}_{G}\left(N_{0}\right)$. Then $N_{0}$ contains $\left|N_{1}: N_{0}\right|>1$ conjugates of $A$ under $N_{1}$. Since $N_{0}$ is nonabelian, the number of abelian subgroups of index $p$ in $N_{0}$ is equal to $p+1$ (see [B, Exercise 1.6(a)]), therefore, we get $\left|N_{1}: N_{0}\right|=p$. The intersection of all abelian subgroups of index $p$ in $N_{0}$ coincides with $\mathrm{Z}\left(N_{0}\right)=Z \triangleleft N_{1}$. The quotient group $N_{1} / Z$ is nonabelian since its subgroup $A / Z$ (of index $p^{2}$ ) is not normal. Since $\mathrm{C}_{G}(A)=A$, we get $\mathrm{Z}(G) \leq Z<A$. Let $R \leq \mathrm{Z}(G)$ be of order $p$. Then either $A / R$ or $N_{0} / R$ is a maximal abelian subgroup of $G / R$ since $N_{1} / R$, having a nonabelian epimorphic image $N_{1} / \mathrm{Z}(G)$, is nonabelian. Clearly, the pair $K / R<G / R$, where $K / R$ is the chosen above a maximal abelian subgroup of $G / R$ containing $A / R$, satisfies $\left|\mathrm{N}_{G / R}(K / R):(K / R)\right|=p$, since $K \in\left\{A, N_{0}\right\}$. Thus, $K / R$ is soft in $G / R$. By induction, there is only one maximal chain connecting $K / R$ and $G / R$ so there is only one maximal chain connection $A$ and $G$. Indeed, it is nothing to prove if $K=A$. If $K>A$, then $K=N_{0}$ so the result also holds since $N_{0}$ is a unique subgroup of $G$ of order $p|A|$ containing $A$. Similarly, by induction, we obtain the second assertion on indices.

REMARK 4. If $R$ and $G$ are as in Theorem 2, then every subgroup $H \leq G$ such that $R<H$ and $\exp (H)=p$, has order $\leq p^{p}$. Indeed, $\left|\mathrm{C}_{H}(R)\right|=p^{2}$ so $H$ is of maximal class ([B, Proposition 1.8]), and now the claim follows from Blackburn's theory of $p$-groups of maximal class (see [B, Theorems 9.5, 9.6]).

Case $p=2$ is considered in the following theorem.

TheOREM 5. Suppose that a nonabelian 2-group $G$ contains a maximal elementary abelian subgroup $R$ of order 4 and $R$ is not normal in $G .^{2}$ Then one of the following holds:

(a) The subgroup $\mathrm{C}_{G}(R)$ has a cyclic subgroup of index 2 (so it is abelian).

(b) The subgroup $\mathrm{C}_{G}(R)=Q \times Z$, where $Q$ is a generalized quaternion group and $|Z|=2 .^{3}$

${ }^{2}$ If $R \triangleleft G$, then $G$ has no normal elementary abelian subgroup of order $2^{3}$; the structure of such $G$ is described in [BJ1, $\S 50]$. Note that a minimal nonmetacyclic group $X$ of order $2^{5}$ satisfies $\left|\Omega_{1}(X)\right|=4$ and $\mathrm{d}(X)=3$; the group $X$ is special.

${ }^{3}$ In that case, $\mathrm{Z}(G)=\Omega_{1}(Q)$ has order 2 since this subgroup is characteristic in $C$. It follows that if $Z=\langle z\rangle$, then $\mathrm{C}_{G}(z)=C$. The 2-groups $G$ containing an involution $x$ 
Proof. Set $C=\mathrm{C}_{G}(R)$; then $\Omega_{1}(C)=R$. Since $R$ is not normal in $G$, the subgroup $C$ has no metacyclic subgroup of order 16 and exponent 4 , by Proposition 1.

If $C$ is abelian, we get case (a) since $C$ has no abelian subgroup of type $(4,4)$ and so $\left|\Omega_{2}(C)\right| \leq 2^{3}$ (see the proof of Theorem 2 ).

Now suppose that $C$ is nonabelian. Then $C$ contains a minimal nonabelian subgroup $A$. Since $\Omega_{1}(A) \leq \Omega_{1}(C)=R$, it follows that $A$ is metacyclic ([BJ1, Lemma 65.1]). Assume that $|A|>8$. Then $R<A$ since $\Omega_{1}(A) \cong \mathrm{E}_{4} \cong R=\Omega_{1}(C)$, so $R=\Omega_{1}(A) \leq \mathrm{Z}(A)$, and we conclude that $A$ has no cyclic subgroup of index 2 (otherwise, $A$ will be abelian). Since, by Proposition 1, $A$ has no metacyclic subgroup of order 16 and exponent 4 , we get a contradiction. Therefore, $|A|=8$. Since $A \neq \mathrm{D}_{8}$, it follows that $A \cong \mathrm{Q}_{8}$. Thus, all minimal nonabelian subgroups of $C$ are isomorphic to $\mathrm{Q}_{8}$. It follows that $C=Q \times Z$, where $Q$ is a generalized quaternion group and $|Z|=2$ ([BJ1, Corollary A.17.3]), and the proof is complete.

Proposition 6 ([GM, Lemma 2.5] for $p>2$ ). Suppose that a $p$-group $G$, that is not a 2-group of maximal class, contains a non-G-invariant maximal elementary abelian subgroup $R$ of order $p^{2}$. Then $G$ has only one normal elementary abelian subgroup of order $p^{2}$, unless $p=2$ and $G$ contains a proper subgroup of order $2^{4}$ that is isomorphic to the group $K \cong \mathrm{D}_{8} * \mathrm{C}_{4}$ of order $16 .^{4}$

Proof. Assume that $E$ and $F$ are distinct $G$-invariant abelian subgroups of type $(p, p)$ in $G$. Since $\mathrm{Z}(G)$ is cyclic, we get $E \cap F=U$, where $U=$ $\Omega_{1}(\mathrm{Z}(G))$ so $|E \cap F|=p$ and the subgroup $H=E F$ has order $p^{3}$, by the product formula. The subgroups $E / U, F / U \leq \mathrm{Z}(G / U)$. If $H$ is abelian, it is elementary, and so $R \not \leq H$. If $H$ is nonabelian, it is either of exponent $p>2$ or isomorphic to $\mathrm{D}_{8}$ (this follows from the description of groups of order $p^{3}$ ). In that case, all noncyclic subgroups of index $p$ in $H$ are normal in $G$ since $H / U \leq \mathrm{Z}(G)$ and $U=\Phi(H)$. It follows that $R \not \leq H$. Write $D=H R$; then $\Omega_{1}(D)=D$ and, since $U=H \cap R$ has order $p$, we get $|D|=p^{4}$, by the product formula. Since $E / U$ and $F / U$ are distinct central subgroups of $G / U$, it follows that $D / U \cong \mathrm{E}_{p^{3}}$ so that $\mathrm{d}(D)=3$ and $\operatorname{cl}(D)=2$.

Suppose that $H$ is abelian. In that case, $\mathrm{C}_{D}(R)$ is of exponent $p$ so it coincides with $R$, by hypothesis, and it follows from [B, Proposition 1.8] that $\operatorname{cl}(D)=3>2$, contrary to the last sentence of the previous paragraph.

Now let $H$ be nonabelian. By [B, Proposition 10.17], $\mathrm{C}_{D}(H) \not H$ since $D$ is not of maximal class, and so $\mathrm{Z}(D)$ has order $p^{2}$. It follows that $\mathrm{Z}(D)$ is cyclic (otherwise, $R<R Z(D) \cong \mathrm{E}_{p^{3}}$, contrary to the hypothesis). In that case, we

such that $\mathrm{C}_{G}(x)=Q \times\langle x\rangle$, where $Q$ is either cyclic or a generalized quaternion group, are described in Janko's papers [Jan1] and [Jan2], respectively (see also [BJ1, $\S 48$, 49]), and these sources contain essential additional information on this case.

${ }^{4}$ Note that all abelian subgroups of type $(2,2)$ are normal in $K$. 
have $p=2$ (if $p>2$, then $D=\Omega_{1}(D)$ is of exponent $p$, a contradiction). It follows that $D \cong \mathrm{D}_{8} * \mathrm{C}_{4}$ has order $2^{4}$ (note that $\mathrm{D}_{8} * \mathrm{C}_{4} \cong \mathrm{Q}_{8} * \mathrm{C}_{4}$ ).

In particular, if, in Proposition $6, p>2$, then $G$ has only one normal abelian subgroup of type $(p, p)$, as asserted in [GM, Lemma 2.5].

Definition 7. A proper subgroup $A$ of a p-group $G$ is said to be generalized soft if, whenever $A \leq H<G$, then $\left|\mathrm{N}_{G}(H): H\right|=p$ (in that case, there is only one maximal chain connecting $A$ and $G$ but the converse is not true).

In the following proposition we consider the $p$-groups containing a subgroup of order $p$ that is, as a rule, generalized soft.

Proposition 8. Suppose that a p-group $G$ contains a subgroup $L$ of order $p$ such that there is only one maximal chain connecting $L$ and $G$. Then one of the following holds:

(a) $G$ is abelian with cyclic subgroup of index $p$.

(b) $G=\left\langle a, b \mid a^{p^{n}}=b^{p}=1, b^{a}=a^{1+p^{n-1}}\right\rangle \cong \mathrm{M}_{p^{n+1}}$ (see $[B$, Theorem 1.2]).

(c) $G$ is a p-group of maximal class. ${ }^{5}$

Proof. Write $N=\mathrm{N}_{G}(L)$; then $N / L$ is cyclic. If $N=G$, we have case (a). Next we assume that $N<G$. If $|N / L|=p$, then $G$ is of maximal class, by [B, Proposition 1.8]. Now assume that $|N / L|>p$. Since $L$ is not $G$-invariant, it is not characteristic in $N$ so $N$ is not cyclic. Let $R=\Omega_{1}(N)$ and $\mathrm{N}_{1}=\mathrm{N}_{G}(R)$. Since $R$ is characteristic in $N$, we get $N<N_{1}$. By hypothesis, $N_{1} / R$ is cyclic. Since $R<N<N_{1}$, it follows that $R=\Omega_{1}\left(N_{1}\right)$ is characteristic in $N_{1}$, and we conclude that $N_{1}=G$. In that case, $G$ possesses a cyclic subgroup of index $p$ so $G \cong \mathrm{M}_{p^{n+1}}$, by [B, Theorem 1.2].

REMARK 9. Below we describe the pairs $L<G$ of 2-groups such that $L \cong \mathrm{E}_{4}, L$ is not $G$-invariant and there is only one maximal chain connecting $L$ with $G$. Write $C=\mathrm{C}_{G}(L)$; then $C<G$. One may assume that $L<C$ (otherwise, $G$ is of maximal class, by [B, Proposition 1.8]). In that case, $C / L>\{1\}$ is cyclic so $C$ is a maximal abelian subgroup of $G$ of rank 2 or 3 . If $|C / L|=2$, then $C \in\left\{\mathrm{E}_{8}, \mathrm{C}_{4} \times \mathrm{C}_{2}\right\}$. Such $G$ are described in [BJ1, $\left.\S 50,77\right]$. Next assume that $|C / L|>2$. Let $\mathrm{d}(C)=3$. Then $T=\Omega_{1}(C) \cong \mathrm{E}_{8}$ is a proper characteristic subgroup in $C$. In that case, $\mathrm{N}_{G}(T) / T>C / T>\{1\}$ is cyclic, by hypothesis, and so $T=\Omega_{1}\left(\mathrm{~N}_{G}(T)\right)$ is characteristic in $\mathrm{N}_{G}(T)$, and we conclude that $\mathrm{N}_{G}(T)=G$ hence $\Omega_{1}(G)=T$. Thus, $G / \Omega_{1}(G)$ is cyclic and $\Omega_{1}(G) \cong \mathrm{E}_{8}$. Then $G$ has a cyclic subgroup of index 4 (such $G$ are described in $[\mathrm{BJ} 1, \S 74])$. Now let $C$ be abelian of rank 2 ; then $L=\Omega_{1}(C)$ so $C$ has a cyclic

\footnotetext{
${ }^{5}$ Not all $p$-groups of maximal class contain such a subgroup as $L$ (for example, an irregular $p$-group $G$ of maximal class, $p>3$, such that $\Omega_{1}(G)$ is abelian of order $p^{p-1}$, has no such subgroup).
} 
subgroup of index 2, by Proposition 1 . In that case, $\mathrm{N}_{G}(L) / L$ is cyclic, by hypothesis. Therefore, it follows from $L<C<\mathrm{N}_{G}(L)$ that $L=\Omega_{1}\left(\mathrm{~N}_{G}(L)\right)$ is characteristic in $\mathrm{N}_{G}(L)$ so $\mathrm{N}_{G}(L)=G$, i.e., $L \triangleleft G$, contrary to the hypothesis.

\section{PROBLEMS}

1. Suppose that a $p$-group $G, p>2$, possesses a maximal elementary abelian subgroup of order $p^{2}$ and $H \leq G$. (i) Is it true that $\mathrm{d}(H) \leq p$ ? (ii) Is it true that $|H|<p^{p+1}$ provided $\exp (H)=p$ ?

2. Suppose that a $p$-group $G, p>2$, possesses a maximal elementary abelian subgroup of order $p^{n}$. Is it true that $G$ has no elementary abelian subgroup of order $p^{1+p^{n-1}}$ ?

3. Study the $p$-groups all of whose minimal nonabelian (so all nonabelian) subgroups are generalized soft.

4. Study the $p$-groups containing a cyclic generalized soft subgroup of order $p^{n}$ (the problem is nontrivial even for $n=2$ ).

ACKNOWLEDGEMENTS.

I am indebted to George Glauberman acquainting me with paper [GM] prior its publication (this note was inspired by $[\mathrm{GM}]$ ) and Zvonimir Janko for useful discussion. I also indebted the referee for a number of constructive remarks.

\section{REFERENCES}

[B] Y. Berkovich, Groups of prime power order. Vol. 1, Walter de Gruyter, Berlin, 2008.

[BJ1] Y. Berkovich and Z. Janko, Groups of prime power order. Vol. 2, Walter de Gruyter, Berlin, 2008.

[BJ2] Y. Berkovich and Z. Janko, Groups of prime power order. Vol. 3, Walter de Gruyter, Berlin, 2011.

[Bla1] N. Blackburn, Generalizations of certain elementary theorems on p-groups, Proc. London Math. Soc. (3) 11 (1961), 1-22.

[Bla2] N. Blackburn, Groups of prime-power order having an abelian centralizer of type $(r, 1)$, Monatsh. Math. 99 (1985), 1-18.

[GM] G. Glauberman and N. Mazza, p-groups with maximal elementary abelian subgroup of rank 2, J. Algebra 323 (2010), 1729-1737.

[H] L. Hethelyi, Some remarks on 2-groups having soft subgroups, Studia Sci. Math. Hungar. 27 (1992), 295-299.

[Jan1] Z. Janko, Finite 2-groups with small centralizer of an involution, J. Algebra 241 (2001), 818-826.

[Jan2] Z. Janko, Finite 2-groups with small centralizer of an involution, 2, J. Algebra 245 (2001), 413-429.

Y. Berkovich

Department of Mathematics

University of Haifa

Mount Carmel, Haifa 31905

Israel

Received: 10.2.2010.

Revised: 4.4.2010. 